\title{
Basic reproduction number and predicted trends of coronavirus disease 2019 epidemic in the mainland of China
}

Yong $\mathrm{Li}^{1+}$, Lian-Wen Wang ${ }^{2 \dagger}$, Zhi-Hang Peng ${ }^{3^{*}}$ and Hong-Bing Shen ${ }^{3^{*}}$

\begin{abstract}
Background: Coronavirus disease 2019 (COVID-19) has caused a serious epidemic around the world, but it has been effectively controlled in the mainland of China. The Chinese government limited the migration of people almost from all walks of life. Medical workers have rushed into Hubei province to fight against the epidemic. Any activity that can increase infection is prohibited. The aim of this study was to confirm that timely lockdown, largescale case-screening and other control measures proposed by the Chinese government were effective to contain the spread of the virus in the mainland of China.
\end{abstract}

Methods: Based on disease transmission-related parameters, this study was designed to predict the trend of COVID-19 epidemic in the mainland of China and provide theoretical basis for current prevention and control. An SEIQR epidemiological model incorporating asymptomatic transmission, short term immunity and imperfect isolation was constructed to evaluate the transmission dynamics of COVID-19 inside and outside of Hubei province. With COVID-19 cases confirmed by the National Health Commission (NHC), the optimal parameters of the model were set by calculating the minimum Chi-square value.

Results: Before the migration to and from Wuhan was cut off, the basic reproduction number in China was 5.6015. From 23 January to 26 January 2020, the basic reproduction number in China was 6.6037. From 27 January to 11 February 2020, the basic reproduction number outside Hubei province dropped below 1, but that in Hubei province remained 3.7732. Because of stricter controlling measures, especially after the initiation of the large-scale case-screening, the epidemic rampancy in Hubei has also been contained. The average basic reproduction number in Hubei province was 3.4094 as of 25 February 2020. We estimated the cumulative number of confirmed cases nationwide was 82 186, and 69230 in Hubei province on 9 April 2020.

Conclusions: The lockdown of Hubei province significantly reduced the basic reproduction number. The large-scale case-screening also showed the effectiveness in the epidemic control. This study provided experiences that could be replicated in other countries suffering from the epidemic. Although the epidemic is subsiding in China, the controlling efforts should not be terminated before May.

Keywords: Coronavirus disease 2019, SEIQR model, Basic reproduction number, Parameter estimation, Lockdown, Large-scale case-screening

\footnotetext{
* Correspondence: zhihangpeng@njmu.edu.cn; hbshen@njmu.edu.cn

${ }^{\dagger}$ Yong Li and Lian-Wen Wang contributed equally to this work.

${ }^{3}$ Department of Epidemiology and Biostatistics, School of Public Health,

Nanjing Medical University, Nanjing 210029, China

Full list of author information is available at the end of the article
}

\section{$\triangle B M C$}

(c) The Author(s). 2020 Open Access This article is licensed under a Creative Commons Attribution 4.0 International License, which permits use, sharing, adaptation, distribution and reproduction in any medium or format, as long as you give appropriate credit to the original author(s) and the source, provide a link to the Creative Commons licence, and indicate if changes were made. The images or other third party material in this article are included in the article's Creative Commons licence, unless indicated otherwise in a credit line to the material. If material is not included in the article's Creative Commons licence and your intended use is not permitted by statutory regulation or exceeds the permitted use, you will need to obtain permission directly from the copyright holder. To view a copy of this licence, visit http://creativecommons.org/licenses/by/4.0/ The Creative Commons Public Domain Dedication waiver (http://creativecommons.org/publicdomain/zero/1.0/) applies to the data made available in this article, unless otherwise stated in a credit line to the data. 


\section{Background}

Coronavirus disease 2019 (COVID-19) is caused by a novel coronavirus, formerly named as 2019-nCoV by World Health Organization (WHO) on 12 January 2020 and then severe acute respiratory syndrome coronavirus 2 (SARS-CoV-2) by the International Committee on Taxonomy of Viruses (ICTV) on 11 February 2020 [1, 2]. The epidemic has spread rapidly across the world [3-5]. In China, a cluster of pneumonia cases was reported in $\mathrm{Wu}-$ han, Hubei province, in December 2019 [6-8]. Rapidly, Hubei province became the hardest-hit of the COVID-19 epidemic, due to the high rate of human-to-human transmission [9-11], mainly through droplets from coughing or sneezing or body contact. Immediately, the Chinese government took drastic controlling efforts (like public education, active surveillance, early detection, case management, contact tracing, especially mandatory quarantine for at least 14 days [12]). As of 26 May 2020 (24:00 GMT + 8), 82993 confirmed cases, 6 suspected cases and 4634 deaths were reported in China, most of them from Hubei province (68 135 confirmed cases and 4512 deaths) [13]. This epidemic is attacking 215 countries and regions around the world, such as USA, Spain, Italy, the United Kingdom, Russian Federation, Germany, Brazil, France, Turkey, Iran, Canada, Peru, India, Belgium, Netherlands and Republic of Korea. There have appeared 5404512 confirmed cases and 343514 deaths worldwide until 26 May 2020 (10:00 GMT + 2) [14].

SARS-CoV-2, the Middle East respiratory syndrome coronavirus (MERS-CoV), and the flu virus belong to the same family and cause similar symptoms [15, 16]. People of all ages are susceptible. Onset manifestations of COVID-19 include fever, cough symptoms, myalgia or fatigue, and a few or few present sputum overproduction, headache, haemoptysis and diarrhea [17-22]. Though showing no symptoms or only mild symptoms, some patients still transmit the virus, a phenomenon that is called asymptomatic transmission [23-26]. No specific treatment drugs have yet been created for COVID-19 [12, 27]. COVID-19 is self-limiting, and patients often heal through their immunity. Many asymptomatic patients can recover after self-quarantine [28].

The 2002-2003 SARS epidemic led to 8096 cases and 774 deaths (mortality 9.6\%) in 29 countries or regions [29], and the persistent MERS epidemics led to 2494 cases and 858 deaths (mortality 34.4\%) in 27 countries during 2012-2019 [30]. The COVID-19 epidemic has aroused global health concern [31]. Many countries implemented mandatory quarantine in spite of its relatively low mortality (2.8\%). For instance, the government of China limited inflow and outflow of people almost from all walks of life, and suspended all kinds of mass activities. But, medical workers have streamed into Hubei province to fight against the epidemic.
Therefore, to find more effective control efforts, the peak arrival time and the trend of the COVID-19 epidemic in the mainland of China should be predicted with a well-designed model [32, 33]. Many studies have estimated the reproduction number in the early phase of COVID-19 outbreak in China [32-43].

This study based on the cumulative confirmed cases, cured and discharged cases, death tolls and suspected cases released from the National Health Commission (NHC) [13]. These findings formulate a SEIQR (susceptible-exposed-infected but not hospitalized-infectious and isolatedrecovered) epidemic model to explore the impacts of the lockdown of Wuhan and the curb of population migration on COVID-19 transmission. The model was constructed, incorporating asymptomatic transmission, short term immunity and imperfect isolation. The aim of this study was to prove that timely lockdown, large-scale case-screening and other control measures proposed by the Chinese government were effective means to contain the spread of the virus in the mainland of China. Through parameter estimation, we got the parameters of the model. With the aid of sensitivity analysis, we evaluated the timeliness and correctness of the early measures implemented by the Chinese government. Before 25 February 2020, we predicted the epidemic trend in China, and the final number of cumulative confirmed cases in Hubei province and the mainland of China. Up to now, the results have been proved accurate. At the same time, the basic reproduction number of each stage is decreasing, which reflects that the control measures of the Chinese government play a decisive role. Before 25 February, we also gave suggestions on the time for full resumption of work. Although the epidemic is under control, it is not the time to terminate the controlling efforts which are expected to be ended as early as in May.

\section{Methods}

\section{The COVID-19 model}

In the mainland of China, the government of China is rigorously limiting the migration of people among all provinces, with Hubei province completely cut off from the outside. For this reason, Hubei province and other provinces are considered into two patches, denoted as 1 and 2, respectively (e.g., seeing [32, 44]). In each patch, the population related to COVID-19 is divided into five epidemiological subgroups: susceptible, $S_{i}$; exposed, $E_{i}$; infected but not hospitalized, $I_{i}$ (including suspected, carrier and undetected); infectious and isolated (daily confirmed real-time cases announced by NHC [13]), $Q_{i}$; and recovered (short term immunity), $R_{i}$. The total population $N_{i}=S_{i}+E_{i}+I_{i}+Q_{i}+R_{i}, i=1,2$. In order to better reflect the actual situation of COVID-19 transmission in the mainland of China, we set the following conditions: 
1) Natural birth and death are ignored since we only focus on the short-term disease transmission;

2) Asymptomatic transmission [23-26] is the one mode of transmission of COVID-19; the susceptible $\left(S_{i}\right)$ individuals may be infected due to contacts with the infected but not hospitalized individuals $\left(I_{i}\right)$. The individuals in incubation period $\left(E_{i}\right)$ also have the potential to transmit the virus. Meanwhile, infectious and isolated individuals $\left(Q_{i}\right)$ also have a certain probability to transmit the virus to medical workers and others, which is a phenomenon called imperfect isolation. Therefore, the exposed $\left(E_{i}\right)$ and the infectious and isolated $\left(Q_{i}\right)$ are considered infectious, with infectivity reduction factors $k_{i}$ and $l_{i}$, respectively;

3) A few infected individuals do not develop obvious symptoms and have no short-term immunity after self-healing [28], so it is assumed that these individuals $\left(\gamma_{i} \eta_{i} I_{i}\right)$ will directly return to the susceptible;

4) Wuhan was locked down off at 10:00 AM, 23 January 2020, and other cities in Hubei province were locked down successively. Xiangyang City in Hubei province was lastly locked down at 00:00 on 27 January 2020. So, the migration rates are considered as follows:

$\omega=\left\{\begin{array}{l}\omega_{a}, 11-22 \text { January 2020, Wuhan was not locked down } \\ \omega_{b}, 23-26 \text { January 2020, Wuhan was locked down } \\ \omega_{c}, \text { After } 26 \text { January 2020, Hubei was locked down }\end{array}\right.$

5) The lockdown of Wuhan city and Hubei province, and the large-scale case-screening starting on 12 February may bring out impacts on the transmission rates $\left(\beta_{i} i=1,2\right)$ among individuals, so

$\beta_{\mathrm{i}}=\left\{\begin{array}{l}\beta_{i a}, 11-22 \text { January 2020, Wuhan was not locked down, } \\ \beta_{i b}, 23-26 \text { January 2020, Wuhan was locked down, } \\ \beta_{i c}, 26 \text { January-11 February 2020, Hubei was locked down, } \\ \beta_{i d}, \text { After 11 February 2020, the large-scale case-screening has begun } .\end{array}\right.$

6) Before the lockdown of Hubei province, we consider that infectivity reduction factors $\left(l_{i}, i=1,2\right)$ between medical workers and patients before 26 January is much higher than that after 26 January, due to the shortage of medical resources and the insufficient understanding of COVID-19

transmission:

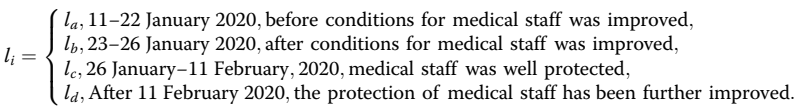

A schematic flow diagram was created for illustrating the transmission dynamics of the COVID-19 infection in Fig. 1. And the biological meanings and acceptable ranges of all parameters are listed in Table 1. The model is described by the following system of ordinary differential equations:

$$
\left\{\begin{array}{l}
\frac{d S_{1}}{d t}=-\beta_{1} S_{1} \frac{\left(I_{1}+k_{1} E_{1}+l_{1} Q_{1}\right)}{N_{1}}-\omega S_{1}+\gamma_{1} \eta_{1} I_{1}, \\
\frac{d E_{1}}{d t}=\beta_{1} S_{1} \frac{\left(I_{1}+k_{1} E_{1}+l_{1} Q_{1}\right)}{N_{1}}-\left(\alpha_{1}+\omega\right) E_{1}, \\
\frac{d I_{1}}{d t}=\alpha_{1}\left(1-\rho_{1}\right) E_{1}-\left(\gamma_{1}+\omega\right) I_{1}, \\
\frac{d Q_{1}}{d t}=\alpha_{1} \rho_{1} E_{1}+\gamma_{1}\left(1-\eta_{1}\right) I_{1}-\left(\delta_{1}+d\right) Q_{1}, \\
\frac{d R_{1}}{d t}=\delta_{1} Q_{1}-\omega R_{1} \\
\frac{d S_{2}}{d t}=-\beta_{2} S_{2} \frac{\left(I_{2}+k_{2} E_{2}+l_{2} Q_{2}\right)}{N_{2}}+\omega S_{1}+\gamma_{2} \eta_{2} I_{2}, \\
\frac{d E_{2}}{d t}=\beta_{2} S_{2} \frac{\left(I_{2}+k_{2} E_{2}+l_{2} Q_{2}\right)}{N_{2}}-\alpha_{2} E_{2}+\omega E_{1}, \\
\frac{d I_{2}}{d t}=\alpha_{2}\left(1-\rho_{2}\right) E_{2}-\gamma_{2} I_{2}+\omega I_{1}, \\
\frac{d Q_{2}}{d t}=\alpha_{2} \rho_{2} E_{2}+\gamma_{2}\left(1-\eta_{2}\right) I_{2}-\left(\delta_{2}+d\right) Q_{2}, \\
\frac{d R_{2}}{d t}=\delta_{2} Q_{2}+\omega R_{1} .
\end{array}\right.
$$

\section{The basic reproduction number $\left(R_{0}\right)$}

The basic reproduction number $\left(R_{0}\right)$ represents the number of infected during the patient's early infectious period (asymptomatic). This threshold may determine whether a disease will die out (if $R_{0}<1$ ) or become epidemic (if $R_{0}>1$ ). As far as the epidemic demonstrates complex dynamics, $R_{0}<1$ is not only the condition guaranteeing that the fate of the disease, but the smaller the better. Following Driessche and Watmough [48], we can compute the basic reproduction number as $R_{0}=\max \{$ $\left.R_{0}{ }^{(1)}, R_{0}{ }^{(2)}\right\}$ before 27 January 2020:

$$
\begin{aligned}
& R_{0}{ }^{(1)}=\underbrace{\frac{\beta_{1} k_{1}}{\alpha_{1}+\omega}}_{\begin{array}{c}
\text { contatc with exposed class } \\
:=R_{01}+R_{02}+R_{03}
\end{array}}+\underbrace{\frac{\beta_{1} \alpha_{1}\left(1-\rho_{1}\right)}{\left(\alpha_{1}+\omega\right)\left(\gamma_{1}+\omega\right)}}_{\text {contact with infected class }}+\underbrace{\frac{\beta_{1} l_{1} \alpha_{1}\left[\rho_{1}\left(\gamma_{1}+\omega\right)+\gamma_{1}\left(1-\rho_{1}\right)\left(1-\eta_{1}\right)\right]}{\left(\alpha_{1}+\omega\right)\left(\gamma_{1}+\omega\right)\left(\delta_{1}+d\right)}}_{\text {contact with isolated class }} \\
& R_{0}^{(2)}=\underbrace{\frac{\omega \beta_{2} k_{2}}{\alpha_{2}\left(\alpha_{1}+\omega\right)}}_{\text {travel with exposed class }}+\underbrace{\frac{\omega \beta_{2}\left[\alpha_{1}\left(1-\rho_{1}\right)+\left(\gamma_{1}+\omega\right)\left(1-\rho_{2}\right)\right]}{\gamma_{2}\left(\alpha_{1}+\omega\right)\left(\gamma_{1}+\omega\right)}}_{\text {travel with infected class }} \\
& +\underbrace{\frac{\omega \beta_{2} l_{2}\left[\alpha_{1}\left(1-\rho_{1}\right)\left(1-\eta_{2}\right)+\left(\gamma_{1}+\omega\right)\left(1-\rho_{2}\right)\left(1-\eta_{2}\right)+\rho_{2}\left(\gamma_{1}+\omega\right)\right]}{\left(\alpha_{1}+\omega\right)\left(\gamma_{1}+\omega\right)\left(\delta_{1}+d\right)\left(\delta_{2}+d\right)}}_{:=R_{04}+R_{05}+R_{06} \quad \text { travel with isolated class }}
\end{aligned}
$$

Here, $R_{01}, R_{02}$ and $R_{03}$ represent the average numbers of the infected individuals by a single exposed individual 


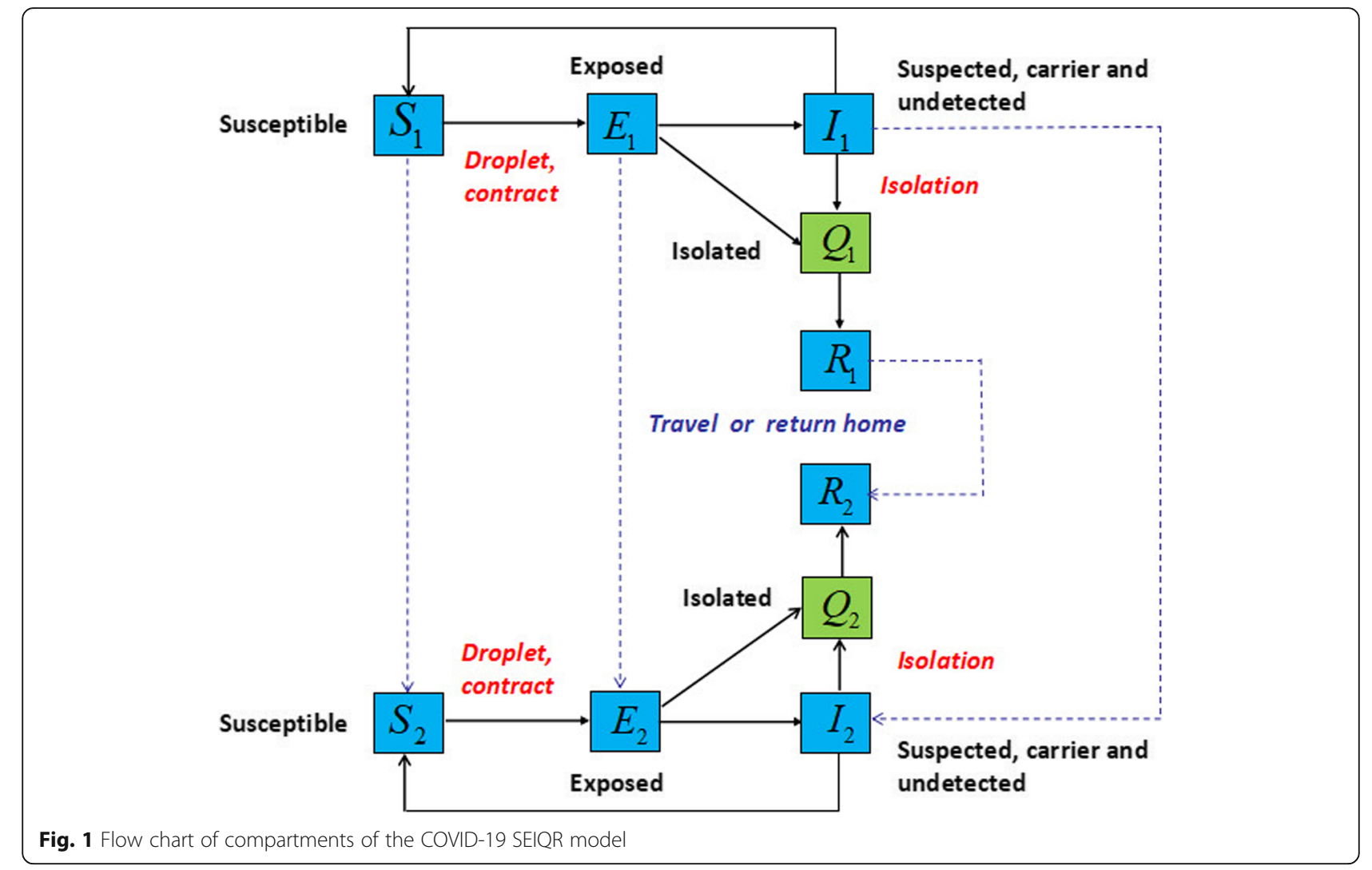

$\left(E_{1}\right)$, infected but not hospitalized individual $\left(I_{1}\right)$ or infectious and isolated individual $\left(Q_{1}\right)$ in a fully susceptible population, respectively. $R_{04}, R_{05}$ and $R_{06}$ represent the average numbers of the infected individuals by a single exposed individual $\left(E_{1}\right.$ and $\left.E_{2}\right)$, infected but not hospitalized individual $\left(I_{1}\right.$ and $\left.I_{2}\right)$ or infectious and isolated individual $\left(Q_{1}\right.$ and $\left.Q_{2}\right)$ in a fully susceptible population travelling to and for, respectively. They represent the contributions of six transmission ways of COVID-19 to the basic reproduction number $R_{0}$.

After 26 January 2020, the whole Hubei province was cut off from the outside, then system (1) is transformed into two independent systems. The basic reproduction number of Hubei province $\left(R_{0}{ }^{(1)}\right)$ and that outside of Hubei $\left(R_{0}{ }^{(2)}\right)$ become

$$
\begin{aligned}
R_{0}(i) & =\frac{\beta_{i} k_{i}}{\alpha_{i}}+\frac{\beta_{i}\left(1-\rho_{i}\right)}{\gamma_{i}}+\frac{\beta_{i} l_{i}\left[\left(1-\rho_{i}\right)\left(1-\eta_{i}\right)+\rho_{i}\right]}{\delta_{i}+d}, i \\
& =1,2 .
\end{aligned}
$$

\section{Parameters estimated}

\section{Data source}

The NHC releases daily reports on cumulative confirmed cases of COVID-19 (positive nucleic acid test result), cured and discharged cases, death tolls and suspected cases of COVID-19 in the mainland of China and Hubei province from 0 to 24 o'clock [13]. On 5 February 2020, NHC released the fifth edition of the Diagnosis and Treatment Protocol for COVID-19 [49]. On 12 February 2020, NHC advocated the large-scale casescreening of previously suspected cases and reexamination of the diagnostic results, and that the diagnosis of COVID-19 should be based on three criteria (previously on two): a history of epidemiological contact or a history of stay in the epidemic area, clinical symptoms (such as fever, cough) and CT features. The NHC announced 59804 confirmed cases nationwide, including 13332 clinically diagnosed cases on 12 February. In the next 2 days, the number rose to 63851 (15384 clinically diagnosed cases) and 66492 (16522 clinically diagnosed cases). With the release of the sixth edition of the Diagnosis and Treatment Protocol for COVID-19 [50], it was officially announced on February 19 that the original two standards were reused for the diagnosis.

By subtracting the data of Hubei province from the national data, we obtained the cumulative case data outside Hubei province. The study involved case data released from 11 January to 25 February 2020. We fitted the daily cumulative confirmed data in Hubei province and those outside Hubei province, and further predicted the number of final confirmed cases. The isolated $Q_{i}(t)$, $i=1,2$, in model (1) represented the daily real-time confirmed cases of Hubei province and outside of Hubei 
Table 1 Parameter estimates for COVID-19 in the mainland of China

\begin{tabular}{|c|c|c|c|c|}
\hline Parameter, initial value & Definition & Value & Standard deviation & Source \\
\hline$\overline{\beta_{1 a} \in[0.01,10]}$ & Transmission rate $\left(\right.$ day $^{-1}$ individual $^{-1}$ ) & 1.0009 & 0.1945 & $\overline{\text { Estimated }}$ \\
\hline$\beta_{1 b} \in[0.01,10]$ & Transmission rate & 1.3754 & 1.076 & Estimated \\
\hline$\beta_{1 c} \in[0.01,10]$ & Transmission rate & 1.0967 & 0.0501 & Estimated \\
\hline$\beta_{1 d} \in[0.01,10]$ & Transmission rate & 0.0809 & 0.0371 & Estimated \\
\hline$\beta_{2 a} \in[0.01,10]$ & Transmission rate & 2.2783 & 1.0798 & Estimated \\
\hline$\beta_{2 b} \in[0.01,10]$ & Transmission rate & 1.4721 & 0.6584 & Estimated \\
\hline$\beta_{2 c} \in[0.01,10]$ & Transmission rate & 0.289 & 0.0176 & Estimated \\
\hline$\beta_{2 d} \in[0.01,10]$ & Transmission rate & 0.0189 & 0.0091 & Estimated \\
\hline$\delta_{1}=\delta_{2} \in[0.0333,0.1]$ & Recovery rate $\left(\right.$ day $\left.^{-1}\right)$ & 0.0618 & 0.0094 & Estimated \\
\hline$\gamma_{1}=\gamma_{2} \in[0.1,0.3333]$ & Detection rate $\left(\right.$ day $\left.^{-1}\right)$ & 0.2269 & 0.0457 & Estimated \\
\hline$\omega_{a} \in[0.0027,0.03]$ & Migration rate $\left(\right.$ day $\left.^{-1}\right)$ & 0.0033 & 0.0002 & Estimated \\
\hline$\omega_{b} \in[0.0027,0.03]$ & Migration rate $\left(\right.$ day $\left.^{-1}\right)$ & 0.0029 & 0.0006 & Estimated \\
\hline$l_{a} \in[0.1,1]$ & Infectivity reduction factor & 0.2001 & 0.0152 & Estimated \\
\hline$I_{b} \in[0.1,1]$ & Infectivity reduction factor & 0.1489 & 0.0164 & Estimated \\
\hline$I_{c} \in[0.1,1]$ & Infectivity reduction factor & 0.0587 & 0.0159 & Estimated \\
\hline$l_{d}$ & Infectivity reduction factor & 0.0001 & - & Fixed \\
\hline$\eta_{1}=\eta_{2} \in[0.01,0.1]$ & Self-healing ratio & 0.0335 & 0.0056 & Estimated \\
\hline$k \in[0.1,1]$ & Infectivity reduction factor $\left(\right.$ day $^{-1}$ ) & 0.3301 & 0.0678 & Estimated \\
\hline$a_{1}=a_{2}$ & Transition rate of exposed $\left(\right.$ day $\left.^{-1}\right)$ & 0.1724 & - & {$[45]$} \\
\hline$\rho_{1}=\rho_{2}$ & Proportion of the infectious & 0.8683 & - & [33] \\
\hline$d$ & Disease-induced death rate $\left(\right.$ day $\left.^{-1}\right)$ & $1.7826 \times 10^{-5}$ & - & [33] \\
\hline$S_{1}(0)$ & Initial susceptible population & $1.10 \times 10^{7}$ & - & {$[46,47]$} \\
\hline$E_{1}(0) \in\left[0,10^{5}\right]$ & Initial exposed population & 12.2544 & 4.3719 & Estimated \\
\hline$I_{1}(0) \in\left[0,10^{5}\right]$ & Initial infected population & 0.1208 & 0.0868 & Estimated \\
\hline$Q_{1}(0)$ & Initial isolated population & 41 & - & Data \\
\hline$R_{1}(0)$ & Initial recovered population & 2 & - & Data \\
\hline$S_{2}(0) \in\left[10^{3}, 1.3 \times 10^{9}\right]$ & Initial susceptible population & $1.23 \times 10^{8}$ & $1.31 \times 10^{5}$ & Estimated \\
\hline$E_{2}(0) \in\left[0,10^{4}\right]$ & Initial exposed population & 0.0184 & 0.0094 & Estimated \\
\hline$I_{2}(0) \in\left[0,10^{5}\right]$ & Initial infected population & 0.0154 & 0.0087 & Estimated \\
\hline$Q_{2}(0)$ & Initial isolated population & 0 & - & Data \\
\hline$R_{2}(0)$ & Initial recovered population & 0 & - & Data \\
\hline
\end{tabular}

- means not applicable

province, respectively. So, the following two equations could describe the dynamics of the cumulative confirmed cases (cumulative isolated) in Hubei province and outside Hubei province, respectively:

$$
\left\{\begin{array}{l}
\frac{d L_{1}}{d t}=\alpha_{1} \rho_{1} E_{1}+\gamma_{1}\left(1-\eta_{1}\right) I_{1}, \\
\frac{d L_{2}}{d t}=\alpha_{2} \rho_{2} E_{2}+\gamma_{2}\left(1-\eta_{2}\right) I_{2} .
\end{array}\right.
$$

\section{Parameter estimation}

From the work of Tang et al. [33], we set the proportion of the infectious $\rho_{1}=\rho_{2}=0.8683$, disease-induced death rate $d=1.7826 \times 10^{-5}$. The susceptible population in
Hubei province was considered as the permanent population in Wuhan city. According to the statistical yearbook data of Wuhan city $[46,47]$, we then assumed $S_{1}(0)=1.1 \times 10^{7}$. According to the cumulative daily case data reported from the NHC [13], we set $Q_{1}(0)=41$, $R_{1}(0)=2, Q_{2}(0)=0, R_{2}(0)=0$. The incubation period $(1 /$ $\left.\alpha_{i}, i=1,2\right)$ of COVID-19 was 5.8 days [45], so transition rate of exposed individuals $E_{i}$ read $\alpha_{1}=\alpha_{2}=0.1724$. We consulted the values of $k$ and $l$ in the literature [51] and got the appropriate range of $k$ and $l$. Considering that the patient's disease course $\left(1 / \delta_{i}, i=1,2\right)$ is about 10 to 30 days, and the time required to detect a suspected patient $\left(1 / \gamma_{i}, i=1,2\right)$ is 3 to 10 days, we thus set the ranges of parameters $\delta_{i}$ and $\gamma_{i}, i=1,2$, respectively. Based on 
$[34,37]$, the range of transmission rate $\beta_{i}, i=1,2$, was given. Before the lockdown of Wuhan, we assume that $30 \%$ (about 300000 ) of the total population in Wuhan had traveled to and back home every day from 11 to 22 January. The average time to move out of Wuhan was 1 day. Therefore, the upper limit of the rate of migration out of Wuhan (all citizens moved out, $\omega$ ) is $0.03 \times 1 / 1=$ 0.03 and the lower limit of the mobility is $1 / 365=$ 0.0027 , considering that everyone moves out of Wuhan at least once a year. The lower and upper limits of other parameters and initial values of model (1) are shown in Table 1.

The detailed steps of simulations were stated as follows.

1) Before closing the city (11 January-22 January 2020): The period is the prophase of high-rate transmission since people do not know that COVID-19 can be transmitted from person to person;

2) After Wuhan was locked down and before Hubei province was locked down (23 January26 January 2020): Wuhan was locked down at 10:00 AM on 23 January, and the last city of Hubei province was locked down (Xiangyang city) in the early morning of 27 January. During this period, the lockdown may bring many sharp impacts.

Therefore, the values of mobility rate $(\omega)$ and transmission rate $\left(\beta_{i}, i=1,2\right)$ vary, see Table 1 for details.

3) After Hubei province was completely locked down, and before the complete case-screening mainly in Hubei province started (23 January11 February 2020): On and after 27 January, all cities in Hubei province were gradually locked down. In this situation, there may be no migration between Hubei province and other provinces, so the migration rate could be fixed as 0 . Supposing that the transmission rate $\left(\beta_{i}\right.$, $i=1,2)$ and the infectivity reduction factors $(l)$ between the medical staff and the patient vary. In addition, with the lockdown of all cities across the mainland of China, Hubei province implemented a more rigorous control. We believed that a large number of susceptible persons $(S)$ were in a relatively safe situation and could not be infected. Therefore, on the 27th day, we assumed that the number of susceptible people (i.e., $S_{1}(17)$ and $S_{2}(17), 27$ January was the 17th day of our simulation) changed greatly due to the lockdown of cities and traffic restrictions throughout the mainland of China, but not in other types of population (i.e., $E, I, Q$ and $R$ ).
4) After large-scale case-screening mainly in Hubei province started (after 11 February 2020): On 12 February, the cumulative confirmed cases announced by the NHC increased by 13332 clinically diagnosed cases in Hubei province. At the same time, large-scale case-screening were carried out nationwide, and stricter control measures were implemented in Hubei province to further restrict residents' move. Therefore, we must assess the impact of the large-scale case-screening that began on 12 February. For other provinces, using the model (1) to reflect the impact of the large-scale casescreening, we assumed that only the transmission rate $\left(\beta_{i}, i=1,2\right)$ was further reduced. Owing to the increasing medical supplies and deepening understanding of the virus, infection in doctors by patients at this time remained extremely rare, so the infectivity reduction factor $(l)$ can be almost ignored $\left(l_{d}=0.0001\right)$ on 12 February (12 February was the 33th day of our simulation). Hence, we used the fewest parameters to characterize the impact of large-scale case-screening on the epidemic. For the confirmed cases of Hubei province on 12 February, it is equivalent to consider that the model has changed its dynamics on that day. Namely, except for the transmission rate $\left(\beta_{1}\right)$ and infectivity reduction factor $(l)$ change, all variables of the model (1) of Hubei province have changed. The changes are analyzed in Section 5.

Hence, we segmented setting basis of parameters (mobility rate $\omega$, transmission rate $\beta_{i}, i=1,2$, and the infectivity reduction factors between the medical staff and the patient $l$ ) in Section 2. The last data in the four stage simulated was collected on 25 February 2020. From 11 January to 25 February 2020, there were 46 confirmed cases in Hubei province and 46 confirmed cases outside Hubei province.

As evidenced by the small cumulative number of confirmed cases in the prophase of the outbreak and large cumulative number of confirmed cases in the metaphase, the numeric curve fluctuates greatly. In order to achieve a better fitting effect, the Chi-square value was chosen to evaluate the reliability of model (1). We estimated the remaining 17 parameters and 5 initial values through calculating the minimum sum of Chi-square $[52,53]$.

$$
J=\sum_{i=1}^{46} \frac{\left(L\left(t_{i}\right)-\hat{L}\left(t_{i}\right)\right)^{2}}{\hat{L}\left(t_{i}\right)}
$$

with the MATLAB (the Mathworks, Inc.). Here, $L\left(t_{i}\right)$, $i=1,2, \cdots, 46$ represent the actual daily confirmed cases, $\hat{L}\left(t_{i}\right), i=1,2, \cdots, 46$ stand for the responding fitting values. The parameter values of model (1) at different 
stages and the initial values of model (1) on 11 January were given in Table 1 .

\section{Results}

Fitting results and analysis of control measures

Cumulative daily cases $\left(L_{1}(\mathrm{t})\right)$ and actual confirmed cases in Hubei province and cumulative daily cases $\left(L_{1}(\mathrm{t})+\right.$ $L_{2}(\mathrm{t})$ ) and actual confirmed cases in the mainland of China are seen in Figs. 2, 4 and 5. According to the description in the previous section, the model (1) has changed its pattern for three times. The simulation results are very consistent with the actual cumulative confirmed cases. Next, we detailed the rationality of these major controlling measures adopted by $\mathrm{NHC}$ and the necessity of adapting our model to a new situation.

1) If Wuhan was not locked down on 23 January, and no subsequent controlling measures were taken: We use the parameters of the first stage (11 January-22 January 2020) and the initial values of the model to fit the cumulative confirmed case data of Hubei province $\left(L_{1}(t)\right)$ and the mainland of China $\left(L_{1}(t)+L_{2}(t)\right)$ (Fig. 3). Cumulative cases in Hubei province will rapidly exceed 2 million within one month. So, it is clear that Wuhan was locked down on 23 January is very influential. After 23 January, the transmission rates $\left(\beta_{1}\right.$ and $\left.\beta_{2}\right)$, migration rates $(\omega)$ and infectivity reduction factor $(l)$ of the model (1) changed, but the quantity of each subgroup of the model (1) did not.

2) If Wuhan was locked down on 23 January, but other cities in the mainland of China did not take controlling efforts: We use the parameters of the first and second stage (11 January-26 January 2020). The initial values of the model to fit the cumulative confirmed case data of Hubei province $\left(L_{1}(\mathrm{t})\right)$ and the mainland of China $\left(L_{1}(\mathrm{t})+L_{2}(\mathrm{t})\right)$ are seen in Fig. 3. Cumulative cases in Hubei province will be close to 1 million within one month. It is worth mentioning that with the lockdown of Wuhan, the transmission rate in overall Hubei has not decreased. However, the lockdown of Hubei is also workable.

3) After Wuhan and Hubei were locked down, other cities in China were also locked down one after another, but the large-scale case-screening after 12 February was not initiated: Since 12 February, NHC decided to add clinically diagnosed cases to confirmed cases, resulting in a "dramatic changes" after 12 February. Since 27 January (after Hubei is completely locked down), the clinically diagnosed cases, instead of the total number within the past 16 days, was reported by NHC every day. We assume that the model switches its pattern on 27 January, then we can get the cumulative confirmed cases of Hubei province and the mainland of China (black dotted line) in Figs. 4 and 5 during a time when large-scale case-screening was not conducted. It can be seen that the experimental numbers are greater than the actual numbers. And we see that due to the large-scale case-screening on 12 February, the system switches its pattern again. The cumulative confirmed cases of Hubei province and the mainland of China (blue dotted line) are shown in Figs. 4 and 5. Based on this conjecture, the cumulative number of confirmed cases nationwide was 82 186, and 69230 in Hubei province on 9 April. This scenario validates the necessity of largescale case-screening.

4) Wuhan locked down, Hubei locked down, largescale case-screening taken: After Hubei province was locked down, we estimated that $S_{1}(17)=3.25 \times 10^{4}$, $S_{2}(17)=1.02 \times 10^{4}$. After the initiation of large-scale case-screening, we estimated that $S_{1}(33)=1.94 \times 10^{3}$, $E_{1}(33)=1.83 \times 10^{3}, \quad I_{1}(33)=4.24 \times 10^{3}, \quad Q_{1}(33)=6.18 \times$ $10^{4}, R_{1}(33)=6.55 \times 10^{4}$. Fitted with Matlab (solid purple lines), we estimated the real cases of Hubei province and the mainland of China (Figs. 4 and 5). The cumulative number of confirmed cases nationwide will be 79633 ,

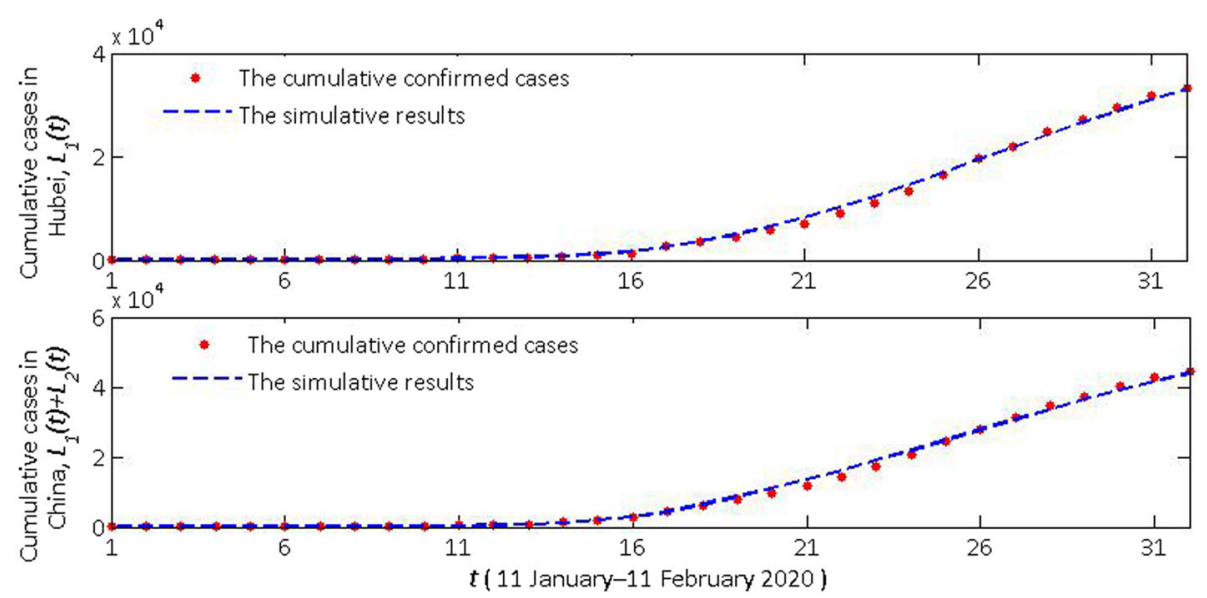

Fig. 2 The cumulative daily confirmed and simulative cases in Hubei province $\left(L_{1}(t)\right)$ and the mainland of China $\left(L_{1}(t)+L_{2}(t)\right)$ 


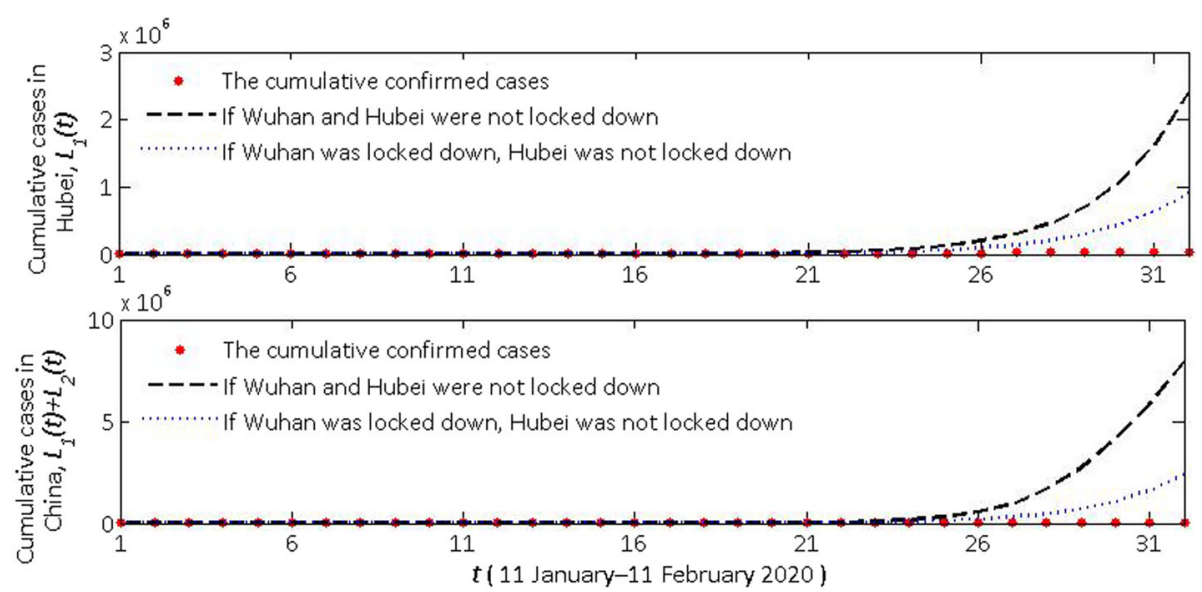

Fig. 3 Comparison of the influence of lockdown of Wuhan and Hubei, China

and 66386 in Hubei province in early March. If the current controlling measures are observed, the cumulative number of cases will gradually reduce in the future.

\section{Results of basic reproduction numbers}

Next, we described the value of the basic reproduction number. From model (1), before Wuhan was locked down (before 23 January), $R_{0}=5.6015$ in the mainland of China; after Wuhan was locked down and before Hubei was locked down (23-26 January), $R_{0}=6.6037$. After Hubei province was locked down and large-scale case-screening was not started (27 January-11 February), the model (1) was divided into two independent models: $R_{0}^{(1)}=3.7732$ in Hubei; $R_{0}{ }^{(2)}=0.9943$ outside Hubei province. After the large-scale case-screening, $R_{0}^{(1)}=0.2020$ in Hubei; $R_{0}{ }^{(2)}=0.0472$ outside Hubei province. We could calculate the basic reproduction number in Hubei province on average as $(5.6015 \times 12+6.6037 \times 4+3.7732 \times$ $16+0.2020 \times 14) / 46=3.4094$ until 25 February.
Sensitivity analysis of basic reproduction number

In order to compare the sensitivity of these parameters to the basic reproduction number in Hubei $\left(R_{0}{ }^{(1)}\right)$ from 27 January to 11 February 2020, we calculated partial rank correlation coefficients (PRCC) with Latin Hypercube Sampling (LHS) [54] to detect the influence of each parameter with uncertain value on $R_{0}{ }^{(1)}$. The sample size was chosen as $n=2000$. We assumed the input parameters were in normal distributions. The expectations (i.e., parameter values) and standard deviations in Table 1. The significance level was chosen as 0.01 . The partial rank correlation coefficients of $R_{0}^{(1)}$ were computed (Table 2). Figure 6 demonstrated its bar chart.

Particularly, the lager absolute value of the PRCC implies greater influence of certain parameter on the change of the cases newly infected with SARS-CoV-2. Thus it could be found that parameters $k, l_{c}, \beta_{1 c}, d$ and $\eta_{1}$ had positive impacts on $R_{0}^{(1)} ; \alpha_{1}, \rho_{1}, \delta_{1}$ and $\gamma_{1}$ had negative impacts. The sensitivity analysis showed that the basic reproduction number was highly sensitive to $\alpha_{1}, k, l_{c}, \rho_{1}, \beta_{1 c}, \delta_{1}$ and $\gamma_{1}$. Therefore, lower transmission

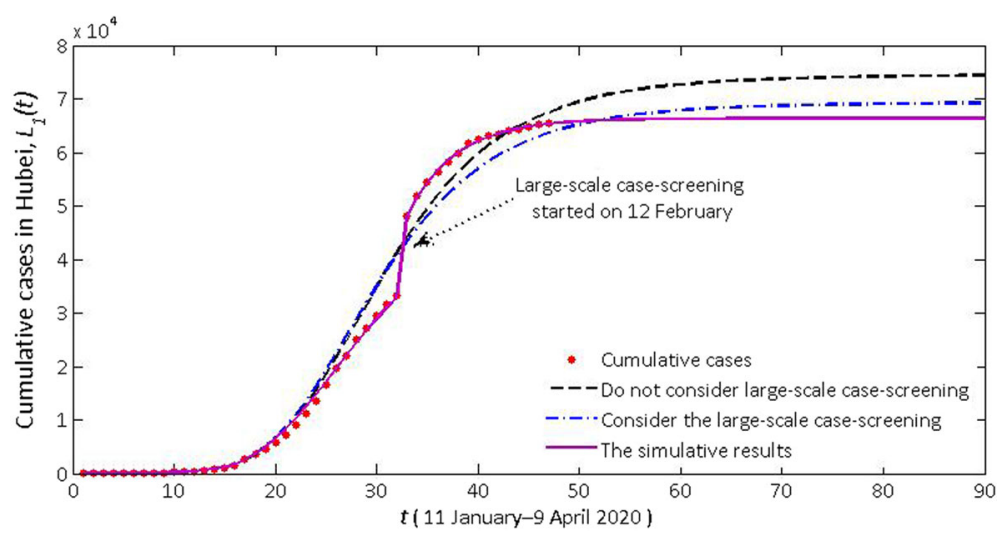

Fig. 4 Impact of the large-scale case-screening (especially in Hubei province) on the number of predicted confirmed cases in Hubei province 


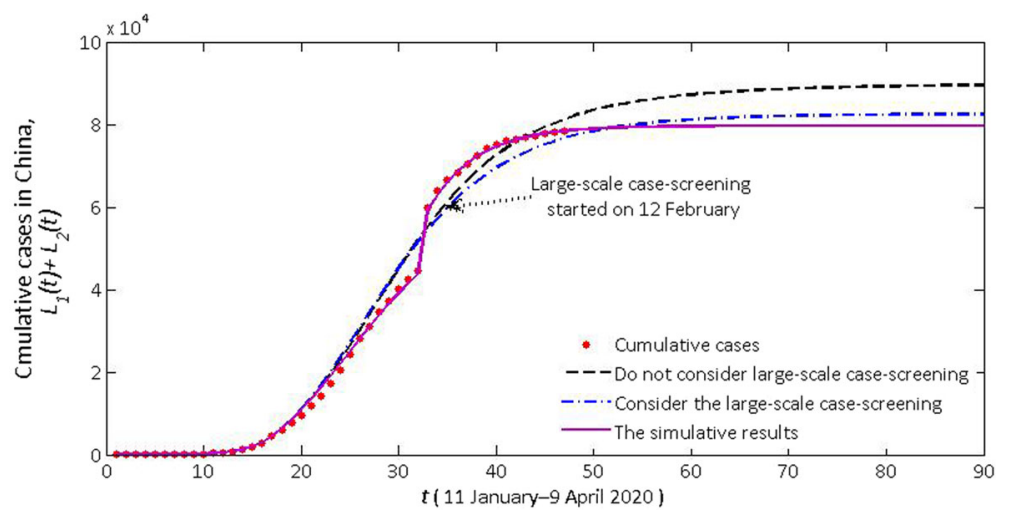

Fig. 5 The number of predicted confirmed cases after the large-scale case-screening started on 12 February (especially in Hubei province) in the mainland of China

rate $(\beta)$, lower infectivity reduction factor $(k$ and $l)$, shorter course of disease $(1 / \delta)$ and higher detection rate $(1 / \gamma)$ could effectively reduce the basic reproduction number.

\section{Discussion}

After the lockdown of Hubei province (around 27 January) and the large-scale case-screening (around 12 February), the transmission rate inside and outside Hubei province decreased significantly. In addition, since the lockdown of Hubei province, the basic reproduction numbers decreased significantly, indicating that the lockdown and large-scale case-screening are effective in controlling the epidemic rampancy across China.

After 27 January, in none-Hubei provinces, the basic reproduction numbers are almost less than one. Under the current conditions, the epidemics outside Hubei province are eventually be controlled, meaning that the cases outside Hubei province are mainly imported. Similarly, the epidemic situation in Hubei province has been basically controlled before and after the start of the large-scale case-screening. From Table $3, R_{01}>R_{02}, R_{03}$ $>R_{02}$ is seen in Hubei province, and $R_{04}>R_{05}, R_{06}>R_{05}$

Table 2 Partial rank correlation coefficients (PRCC) values for $R_{0}^{(1)}$

\begin{tabular}{lll}
\hline Input parameter & PRCC & $P$-value \\
\hline$a_{1}$ & -0.864972677 & 0 \\
$k$ & 0.860166592 & 0 \\
$I_{c}$ & 0.734582947 & 0 \\
$\rho_{1}$ & -0.658402698 & 0 \\
$\beta_{1 c}$ & 0.550790936 & 0 \\
$\delta_{1}$ & -0.50823884 & 0 \\
$\gamma_{1}$ & -0.460646049 & 0 \\
$d$ & 0.043493277 & 0.052272305 \\
$\eta_{1}$ & 0.004758291 & 0.831920484 \\
\hline
\end{tabular}

in the other provinces, showing that close contact between susceptible people $(S)$ and incubation patients $(E)$, and medical staff $(S)$ and isolated patients $(Q)$ is the main route of transmission. The contact between susceptible individuals and suspected, carrier or undetected individuals in model (1) (uniformly defined as infected but not hospitalized individuals with asymptomatic transmission) is not the main route of transmission. Although the transmission rate between susceptible individuals and non-hospitalized patients is the biggest than that in $E$ and $Q$ classes, and the incubation period $(1 / \alpha$, average: 5.8 days $)$ and treatment period $(1 / \delta$, average: 16.18 days) are longer than the detection time $(1 / \gamma$, average: 4.41 days). This may be explained by the larger number of exposed patients $(E)$ than those who are not hospitalized $(I)$.

From Table 3, in the prophase of epidemic (11 January-26 January), the basic reproduction numbers in the mainland of China $\left(R_{0}\right)$ were 5.6015 and 6.6037. These results kept consistent with those of Tang et al. (6.47 [95\% CI: 5.71-7.23]) [33], Shen et al., (4.71 [95\% CI: 4.50-4.92]) [34] and Jia et al. (5.6870 inside Hubei, 6.0295 outside Hubei) [32]. In the metaphase of the epidemic (27 January-11 February), the basic reproduction number in Hubei was 3.7732, the average basic reproduction number was 3.4094 until 25 February. These results agreed with those of Zhao et al. (3.58 [95\% CI: 2.89-4.39]) [35], Imai (1.5-3.5) [55], Read et al. (3.11 [95\% CI: 2.39-4.13]) [56], Cao (4.08) [36], Abbott et al. (2.8-3.8) [37], Bedfordet et al. (1.8-3.5) [19], Chen et al. (2.30 from reservoir to person and 3.58 from person to person) [38] and Huang et al. (3.04-4.35) [39]. More research results argued that the basic reproduction number was less than 3, such as Liu et al. (2.92 [95\% CI: 2.28-3.67]) [15], Li et al. (2.2 [95\% CI: 1.4-3.9]) [18], Riou (2.2) [40], Majumder et al. (2.55) [41], Tang et al. (1.48-1.69 in Xi'an) [42], Zhang et al. (2.28 [95\% CI: 2.06-2.52]) [57], Du et al. (1.32 [95\% CI: 1.16-1.48]) [43] 


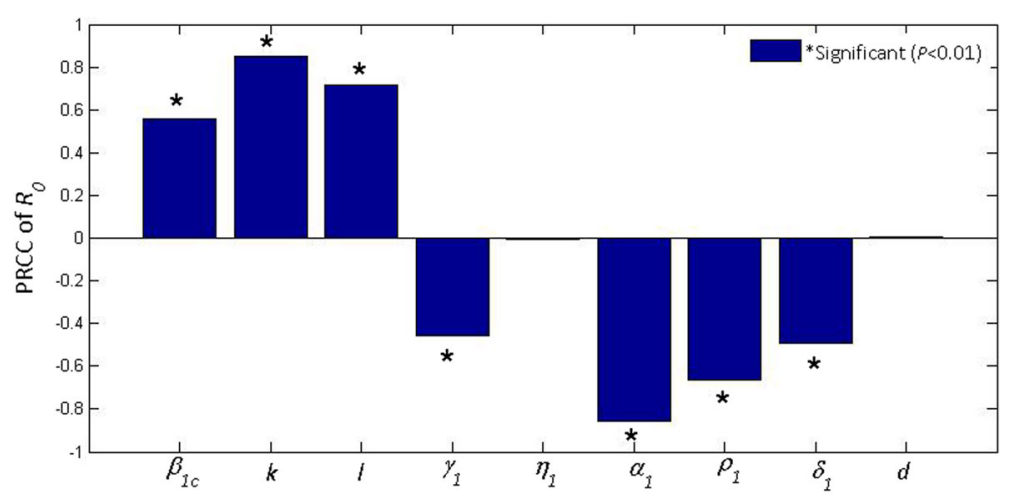

Fig. 6 The values of (PRCC) on the outcome of $R_{0}{ }^{(1)}$. All parameter values were derived from 27 January to 11 February 2020

and so on. In fact, the basic reproduction number is closely related to time and region, and can reflect the severity of the epidemic.

In particular, the number of basic reproduction number in Hubei province was larger than that outside Hubei province. The number of basic reproduction number before the start of large-scale case-screening (prophase and metaphase of the epidemic) was much greater than that after the large-scale case-screening (anaphase of the epidemic). These results were consistent with the conclusion of Jia et al. [32], and this suggested that the epidemic in Hubei province was much more serious.

It also could be seen that our estimated basic reproduction number before 11 February 2020 was slightly higher than that of some previous studies [15, $18,41-43,57]$. This might be mainly caused by the following two reasons. (1) When only Wuhan had confirmed cases at the prophase of the epidemic, we assumed that the number of susceptible individuals on 11 January was the permanent population of Wuhan. With the frequent migration of the population, the epidemic gradually spread across Hubei and China, so there was an increase in the number of susceptible persons before the lockdown of Hubei. But to simplify the discussion of the model, we have omitted this detail. (2) In addition, due to the existence a certain number of asymptomatic infections, the actual number of infections would exceed the confirmed case number released by NHC.

We predicted the impact of the future migration in Hubei province on the epidemic status (see Fig. 7). Once the migration restarts, an increase will be observed in the number of susceptible persons and the transmission rate $\left(\beta_{1}\right)$. We assume that the number of susceptible persons in Hubei will mutate to 10 million. Next, we predicted the cumulative confirmed case at three time points (12 March, 12 April, and 12 May). We only predicted cumulative confirmed case data in the next 2 months. The transmission rates $\left(\beta_{1}\right)$ will be $0.6,0.65$ and 0.7 . It is clear that even with low-level migration (only one million people are susceptible), protective efforts are still needed (the transmission rate is lower than the value of model (1) between January 11 and February 12). Once the migration starts on $12 \operatorname{March}\left(R_{0}{ }^{(1)}=1.4981\right)$, the epidemic situation will rise rapidly. If not controlled, the cumulative number of confirmed diagnoses in Hubei province after two natural months will exceed 71500 . If the migration starts on 12 April $\left(R_{0}^{(1)}=1.6229\right)$ or 12 May $\left(R_{0}{ }^{(1)}=1.7477\right)$, even if the personal protection is

Table 3 The basic reproduction numbers of COVID-19 model (1)

\begin{tabular}{llll}
\hline $11-22$ January & $23-26$ January & 27 January- 11 February & After 12 February \\
\hline$R_{01}=1.8805$ & $R_{01}=2.5900$ & $R_{01}=2.0999$ & $R_{01}=0.1594$ \\
$R_{02}=0.5619$ & $R_{02}=0.7752$ & $R_{02}=0.6366$ & $R_{02}=0.0470$ \\
$R_{03}=3.1592$ & $R_{03}=3.2385$ & $R_{03}=1.0368$ & $R_{03}=1.3 \times 10^{-4}$ \\
$R_{04}=0.0891$ & $R_{04}=0.0466$ & $R_{04}=0.5534$ & $R_{04}=0.0362$ \\
$R_{05}=0.0434$ & $R_{05}=0.0240$ & $R_{05}=0.1677$ & $R_{05}=0.0110$ \\
$R_{06}=2.4444$ & $R_{06}=1.0353$ & $R_{06}=0.2732$ & $R_{06}=3.1 \times 10^{-5}$ \\
$R_{0}^{(1)}=5.6015$ & $R_{0}^{(1)}=6.6037$ & $R_{0}{ }^{(1)}=3.7732$ & $R_{0}^{(1)}=0.2020$ \\
$R_{0}^{(2)}=2.5697$ & $R_{0}^{(2)}=1.1067$ & $R_{0}^{(2)}=0.9943$ & $R_{0}^{(2)}=0.0472$ \\
$R_{0}=5.6015$ & $R_{0}=6.6037$ & - & - \\
\hline
\end{tabular}




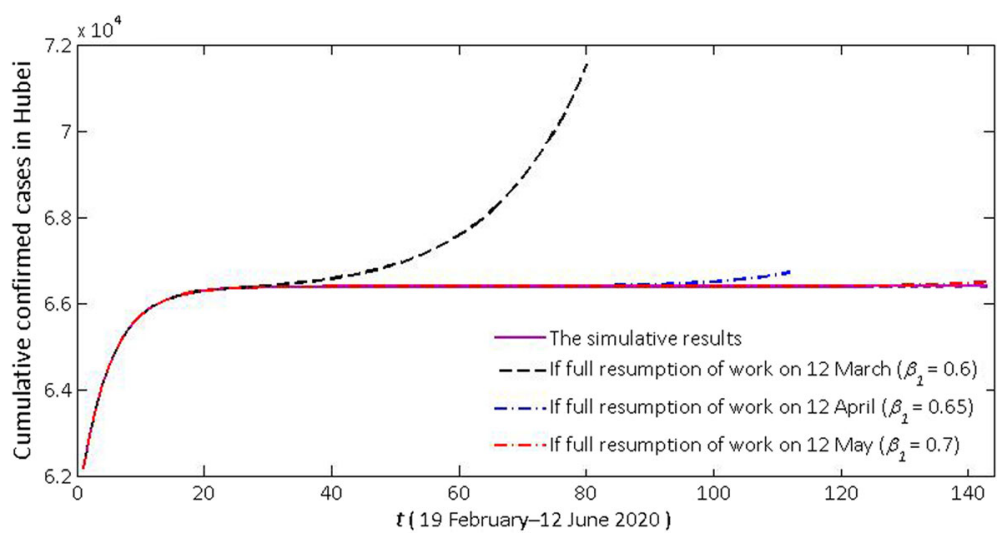

Fig. 7 Effect of future migration (12 March, 12 April or 12 May) on the number of confirmed cases

slightly loose (the transmission rate increases in order), the epidemic seriousness will change relatively little within the next two natural months. Therefore, the current controlling efforts should not be eliminated too earlier. After the epidemic rampancy is completely controlled, theoretically, as long as sufficient precautions are taken (the transmission rate $\beta_{1}$ is less than 0.4005 and the basic reproduction number is just less than 1), the epidemic situation will not break out again.

We obtained the research results on 25 February 2020, a day on which the epidemic in China was not completely quelled, and the global pandemic had not taken shape. At this stage, people cared about the time of the inflection point, the final number of cumulative confirmed cases, the time when the epidemic ended and normal daily activity resumed. In the current global pandemic, China is facing up with imported cases, asymptomatic infections, reinfection of confirmed patients and so on. The Chinese government has issued a series of countering measures, but we have not evaluated them and their impacts on future COVID-19 control, which will be our new research topic.

\section{Conclusions}

China has curbed the spread of COVID-19 epidemic. Hubei province was the worst-hit area in China, especially its Wuhan. The lockdown of Hubei province resulted in a significant reduction in the basic reproduction number. The large-scale case-screening also shows the effectiveness in the epidemic control. The restart of population migration may bring with a risk of second outbreak. This shows that COVID-19 can be fundamentally controlled till its extinction. Although the epidemic is subsiding in China, the controlling efforts should not be terminated before May. This might provide experiences that can be replicated by other countries suffering from the pandemic.

\section{Abbreviations}

WHO: World Health Organization; COVID-19: Coronavirus disease 2019; SARSCoV-2: Severe acute respiratory syndrome coronavirus 2; NHC: National Health Commission; GMT: Greenwich Mean Time; SARS-CoV: Severe acute respiratory syndrome coronavirus; MERS-CoV: Middle East respiratory syndrome coronavirus; SEIQR: Susceptible-exposed-infected but not hospitalized-infectious and isolated-recovered; CT: Computed tomography

\section{Acknowledgments}

We would like to thank anonymous reviewers for very helpful suggestions which improved greatly this manuscript.

\section{Authors' contributions}

LY, WL and PZ participated in study conception and design, collection data; LY and WL performed the mathematical analyses and statistical analyses; LY and WL wrote the manuscript. LY, PZ and SH carried out the initial analyses, reviewed and revised the manuscript; All authors contributed to the interpretation of the results, revised the manuscript critically and approved the final version of the manuscript.

\section{Funding}

The work was partially supported by the National Natural Science Foundation of China (No. 81673275, 11901059), National S\&T Major Project Foundation of China (No. 2017ZX10201101 and 2018ZX10715002), Natural Science Foundation of Hubei province, China (No. 2019CFB353 and 2019CFB241)

\section{Availability of data and materials}

The data that support the findings of this study are available from the National Health Commission (NHC) (http://www.nhc.gov.cn/xcs/yqtb/list_ gzbd.shtml), these network direct data are completely open, and we count these data day by day.

Ethics approval and consent to participate Not applicable.

\section{Consent for publication}

Not applicable.

\section{Competing interests}

The authors declare that there is no conflict of interests regarding the publication of this article. No authors have potential conflicts of interest with reference to this work.

\section{Author details}

'School of Information and Mathematics, Yangtze University, Jingzhou 434023, China. ${ }^{2}$ Department of Mathematics, Hubei Minzu University, Enshi 445000, China. ${ }^{3}$ Department of Epidemiology and Biostatistics, School of Public Health, Nanjing Medical University, Nanjing 210029, China. 
Received: 6 March 2020 Accepted: 18 June 2020

\section{Published online: 16 July 2020}

\section{References}

1. World Health Organization. Naming the coronavirus disease (COVID-19) and the virus that causes it. https://www.who.int/emergencies/diseases/novelcoronavirus-2019/technical-guidance/naming-the-coronavirus-disease(covid-2019)-and-the-virus-that-causes-it. Accessed 12 May 2020.

2. Li R, Pei S, Chen B, Song Y, Zhang T, Yang W, et al. Substantial undocumented infection facilitates the rapid dissemination of novel coronavirus (SARS-CoV2). Science, 2020:368(6490):489-93.

3. Heymann DL, Shindo N. COVID-19: what is next for public health? Lancet. 2020;395(10224):542-5.

4. Gilbert M, Pullano G, Pinotti F, Valdano E, Poletto C, Bölle PY, et al. Preparedness and vulnerability of African countries against importations of COVID-19: a modelling study. Lancet. 2020;395(10227):871-7.

5. Boldog P, Tekeli T, Vizi Z, Dénes A, Bartha FA, Röst G. Risk assessment of novel coronavirus COVID-19 outbreaks outside China. J Clin Med. 2020;9(2): 571.

6. Huang C, Wang Y, Li X, Ren L, Zhao J, Hu Y, et al. Clinical features of patients infected with 2019 novel coronavirus in Wuhan, China. Lancet. 2020:395(10223):497-506.

7. Rong $X$, Yang L, Chu H, Fan M. Effect of delay in diagnosis on transmission of COVID-19. Math Biosci Eng. 2020;17(3):2725-40.

8. Peng L, Yang W, Zhang D, Zhuge C, Hong L. Epidemic analysis of COVID-19 in China by dynamical modeling. arXiv: 2002.06563.

9. Du Z, Wang L, Cauchemez S, Xu X, Wang X, Cowling B, et al. Risk for transportation of 2019 novel coronavirus disease from Wuhan to other cities in China. Emerg Infect Dis. 2020;26(5):1049-52.

10. Chan JF, Yuan S, Kok KH, To KK, Chu H, Yang J, et al. A familial cluster of pneumonia associated with the 2019 novel coronavirus indicating personto-person transmission: a study of a family cluster. Lancet. 2020;395(10223): 514-23.

11. Wu Z, McGoogan JM. Characteristics of and important lessons from the coronavirus disease 2019 (COVID-19) outbreak in China: summary of a report of 72,314 cases from the Chinese Center for Disease Control and Prevention. JAMA. 2020:323(13):1239-42.

12. Bureau of disease prevention and control. Notice on strengthening community prevention and control about COVID-19. http://www.nhc.gov. cn/jkj/s3577/202001/dd1e502534004a8d88b6a10f329a3369.shtml. Accessed 25 Jan 2020.

13. National Health Commission (NHC). Outbreak Notification. http://www.nhc gov.cn/xcs/yqtb/list_gzbd.shtml. Accessed 22 May 2020.

14. World Health Organization. Coronavirus disease (COVID-2019) situation reports. https://www.who.int/emergencies/diseases/novel-coronavirus-2019/ situation-reports/. Accessed 22 May 2020.

15. Liu T, Hu J, Kang M, Lin L, Zhong H, Xiao J, et al. Transmission dynamics of 2019 novel coronavirus (2019-nCoV). bioRxiv. 2020. https://doi.org/10.1101/ 2020.01.25.919787

16. Wu P, Hao X, Lau EH, Wong JY, Leung KS, Wu JT, et al. Real-time tentative assessment of the epidemiological characteristics of novel coronavirus infections in Wuhan, China, as at 22 January 2020. Euro Surveill. 2020;25(3): 2000044.

17. Bogoch II, Watts A, Thomas-Bachli A, Huber C, Kraemer MUG, Khan K. Pneumonia of unknown etiology in Wuhan, China: potential for international spread via commercial air travel. J Travel Med. 2020;27(2):1-3.

18. Li Q, Guan X, Wu P, Wang X, Zhou L, Tong Y, et al. Early transmission dynamics in Wuhan, China, of novel coronavirus-infected pneumonia. New Engl J Med. 2020;382:1199-207.

19. Bedford T, Neher R, Hadfield J, Hodcroft E, Ilcisin M, Müller N. Genomic analysis of nCoV spread. Situation report 2020-01-30. https://nextstrain.org/ narratives/ncov/sit-rep/en/2020-01-30. Accessed 30 Jan 2020.

20. Cohen J, Normile D. New SARS-like virus in China triggers alarm. Science. 2020;367(6475):234-5.

21. Guan W, Ni Z, Hu Y, Liang W, Ou C, He J, et al. Clinical characteristics of coronavirus disease 2019 in China. New Engl J Med. 2020;382:1708-20.

22. Wu JT, Leung $\mathrm{K}$, Leung GM. Nowcasting and forecasting the potential domestic and international spread of the 2019-ncov outbreak originating in Wuhan, China: a modelling study. Lancet. 2020; 2020(10225):689-97.
23. Medical News Today. COVID-19: Study estimates rate of 'silent transmission'. https://www.medicalnewstoday.com/articles/covid-19-study-estimates-rateof-silent-transmission. Accessed 17 Mar 2020.

24. Cai J, Sun W, Huang J, Gamber M, Wu J, He G. Indirect virus transmission in cluster of COVID-19 cases, Wenzhou, China, 2020. Emerg Infect Dis. 2020; 26(6):1343-5

25. USA TODAY. How coronavirus spreads so quickly and how you can slow it down. https://www.usatoday.com/pages/interactives/news/ coronavirus-covid-spread-quickly-how-to-slow-it-down/. Accessed $22 \mathrm{Mar}$ 2020.

26. UpToDate. Coronavirus disease 2019 (COVID-19). https://www.uptodate. com/contents/coronavirus-disease-2019-covid-19? source=history_widget. Accessed 24 Mar 2020

27. Coronavirus medicine. The vaccines and drugs in development to treat COVID-19. https://www.cnet.com/how-to/coronavirus-medicine-thevaccines-and-drugs-in-development-to-treat-covid-19/. Accessed $24 \mathrm{Mar}$ 2020

28. Patient. COVID-19: how to treat coronavirus at home. https://patient.info/ news-and-features/covid-19-how-to-treat-coronavirus-at-home. Accessed 18 Mar 2020.

29. World Health Organization. Summary of probable SARS cases with onset of illness from 1 November 2002 to 31 July 2003. https://www.who.int/csr/ sars/country/table2004_04_21/en/. Accessed 31 Dec 2003.

30. World Health Organization. Middle East Respiratory Syndrome Coronavirus (MERS-CoV). https://www.who.int/emergencies/mers-cov/en/. Accessed 31 Dec 2019.

31. Wang C, Horby PW, Hayden FG, Gao GF. A novel coronavirus outbreak of global health concern. Lancet. 2020;395(10223):470-3.

32. Jia J, Ding J, Liu S, Liao G, Li J, Duan B, et al. Modeling the control of COVID-19: impact of policy interventions and meteorological factors. Electr Differential Equat. 2020;23:1-24.

33. Tang $B$, Wang $X, L i$ Q, Bragazzi NL, Tang S, Xiao Y, et al. Estimation of the transmission risk of the 2019-nCoV and its implication for public health interventions. J Clin Med. 2020;9(2):462.

34. Shen M, Peng Z, Xiao Y, Zhang L. Modelling the epidemic trend of the 2019 novel coronavirus outbreak in China. bioRxiv. 2020. https://doi.org/10.1101/ 2020.01.23.916726

35. Zhao S, Lin Q, Ran J, Musa SS, Yang G, Wang W, et al. Preliminary estimation of the basic reproduction number of novel coronavirus (2019-nCoV) in China, from 2019 to 2020: a data-driven analysis in the early phase of the outbreak. Int J Infect Dis. 2020;92:214-7.

36. Cao Z, Zhang Q, Lu X, Pfeiffer D, Jia Z, Song H, et al. Estimating the effective reproduction number of the 2019-nCoV in China. medRxiv. 2020. https://doi org/10.1101/2020.01.27.20018952

37. Abbott S, Hellewell J, Munday J, CMMID nCoV working group, Funk S The transmissibility of novel coronavirus in the early stages of the 2019-20 outbreak in Wuhan: exploring initial point-source exposure sizes and durations using scenario analysis. Wellcome Open Res. 2020; 5:17.

38. Chen T, Rui J, Wang Q, Zhao Z, Cui J, Yin L. A mathematical model for simulating the phase-based transmissibility of a novel coronavirus. Infect Dis Poverty. 2020;9(1):24.

39. Huang S, Peng Z, Jin Z. Studies of the strategies for controlling the COVID19 epidemic in China: estimation of control efficacy and suggestions for policy makers (in Chinese). Sci Sin Math. 2020:50:1-14.

40. Riou J, Althaus CL. Pattern of early human-to-human transmission of Wuhan 2019 novel coronavirus (2019-nCoV), December 2019 to January 2020. Euro Surveill. 2020;25(4):2000058.

41. Majumder M, Mandl KD. Early transmissibility assessment of a novel coronavirus in Wuhan, China. Available at SSRN. https://doi.org/10.2139/ssin. 3524675.

42. Tang S, Tang B, Bragazzi NL, Xia F, Li T, He S, et al. Stochastic discrete epidemic modeling of COVID-19 transmission in the Province of Shaanxi incorporating public health intervention and case importation. medRxiv. 2020. https://doi.org/10.1101/2020.02.25.20027615.

43. Du Z, Xu X, Wu Y, Wang L, Cowling BJ, Meyers LA. Serial interval of COVID19 among publicly reported confirmed cases. Emerg Infect Dis. 2020;26(6): $1341-3$.

44. Kucharski AJ, Russell TW, Diamond C. Early dynamics of transmission and control of 2019-nCoV: a mathematical modelling study. Lancet Infect Dis. 2020;20(5):553-8. 
45. Backer JA, Klinkenberg D, Wallinga J. Incubation period of 2019 novel coronavirus (2019-nCoV) infections among travellers from Wuhan, China 20-28 January 2020. Euro Surveill. 2020;25(5):2000062.

46. Wuhan Municipal People's Government, Wuhan's resident population exceeds 11 million. http://www.wh.gov.cn/2019_web/whyw/201910/t20191 021_280212.html. Accessed 26 Mar 2019.

47. Wuhan Municipal Bureau of Statistics. The 10th Report of Wuhan's Economic and Social Development Achievements on the 70th Anniversary of the Founding of New China,. http://tjj.wuhan.gov.cn/details.aspx?id=4506. Accessed 29 Sept 2019.

48. Van den Driessche P, Watmough J. Reproduction numbers and subthreshold endemic equilibria for compartmental models of disease transmission. Math Biosci. 2002;180(1-2):29-48.

49. Medical Administration Bureau. Notice on printing and distributing Diagnosis and Treatment Protocol for COVID-19 (trial version 5). http://www. nhc.gov.cn/yzygj/s7653p/202002/3b09b894ac9b4204a79db5b8912d4440. shtml. Accessed 5 Jan 2020.

50. Medical Administration Bureau. Interpretation of Diagnosis and Treatment Protocol for COVID-19 (trial version 6). http://www.nhc.gov.cn/yzygj/s7652 m/202002/54e1ad5c2aac45c19eb541799bf637e9.shtml. Accessed 19 Jan 2020.

51. Chowell G, Fenimore PW, Castillo-Garsow MA, Castillo-Chavez C. SARS outbreaks in Ontario, Hong Kong and Singapore: the role of diagnosis and isolation as a control mechanism. J Theor Biol. 2003;224(1):1-8.

52. Zhang $X$, Zhao $Y$, Neumann AU. Partial immunity and vaccination for influenza. J Comput Biol. 2010;17(12):1689-96.

53. Li Y, Wang L, Pang L, Liu S. The data fitting and optimal control of a hand, foot and mouth disease (HFMD) model with stage structure. Appl Math Comput. 2016;276:61-74

54. Marino S, Hogue IB, Ray CJ, Kirschner DE. A methodology for performing global uncertainty and sensitivity analysis in systems biology. J Theor Biol. 2008;254(1):178-96.

55. Imai N, Cori A, Dorigatti I, Baguelin M, Donnelly CA, Riley S, et al. Report 3: transmissibility of 2019-nCov. https://www.imperial.ac.uk/media/ imperialcollege/medicine/sph/ide/gida-fellowships/Imperial-2019nCoVtransmissibility.pdf. Accessed 25 Jan 2019.

56. Read JM, Bridgen JR, Cummings DA, Ho A, Jewell CP. Novel coronavirus 2019-nCoV: early estimation of epidemiological parameters and epidemic predictions. medRxiv. 2020. https://doi.org/10.1101/2020.01.23.20018549.

57. Zhang S, Diao M, Yu W, Pei L, Lin Z, Chen D. Estimation of the reproductive number of novel coronavirus (COVID-19) and the probable outbreak size on the Diamond princess cruise ship: a data-driven analysis. Int J Infect Dis. 2020;93:201-4

Ready to submit your research? Choose BMC and benefit from:

- fast, convenient online submission

- thorough peer review by experienced researchers in your field

- rapid publication on acceptance

- support for research data, including large and complex data types

- gold Open Access which fosters wider collaboration and increased citations

- maximum visibility for your research: over $100 \mathrm{M}$ website views per year

At $\mathrm{BMC}$, research is always in progress.

Learn more biomedcentral.com/submissions 